\title{
Perceived Chronic Social Adversity, Perceived Social Support, and Depression Among Chinese College Students: Mediating Role of Self-esteem
}

\author{
Tong Jin ${ }^{1, ~ *, ~ X i n x i n ~} \mathrm{Zhu}^{2}$ \\ ${ }^{1}$ Department of Social Work, Beijing College of Social Administration, Beijing, China \\ ${ }^{2}$ Department of Psychology, The University of Edinburgh, Edinburgh, the UK \\ Email address: \\ jt_jintong@126.com (Tong Jin), X.Zhu-54@sms.ed.ac.uk (Xinxin Zhu) \\ ${ }^{*}$ Corresponding author
}

To cite this article:

Tong Jin, Xinxin Zhu. Perceived Chronic Social Adversity, Perceived Social Support, and Depression Among Chinese College Students: Mediating Role of Self-esteem. American Journal of Applied Psychology. Vol. 11, No. 1, 2022, pp. 1-8. doi: 10.11648/j.ajap.20221101.11

Received: December 12, 2021; Accepted: January 4, 2022; Published: January 12, 2022

\begin{abstract}
With the development of society, people pay more attention to mental health. Meanwhile, depression is becoming a major psychological problem faced by Chinese youth. We examined the mediational roles of self-esteem in accounting for the relation between perceived chronic social adversity and depression and the relation between perceived social support and depression among Chinese college students. A total of 805 Chinese college students ( $65.6 \%$ females, mean age $=20.17$ years) completed a multi-measure questionnaire. A cross-sectional correlational research design was used in this study. Obtained data were analyzed using correlational and mediation analysis by Hayes (PROCESS macro). Results of the correlational analysis indicate that perceived chronic social adversity was significantly positively related to depression, but perceived social support was found significantly negatively correlated with depression among Chinese college students. PROCESS macro was used to test the mediating role of self-esteem based on proposed mediation model. The results of mediation analysis revealed that: (1) perceived chronic social adversity positively related to depression, whereas perceived social support negatively related to depression, (2) self-esteem partially mediated the relation between perceived chronic social adversity and depression, and (3) self-esteem entirely mediated the relation between perceived social support and depression among Chinese college students. The results suggest that college students' depression can improve by positive cognition of social encounters via obtaining a high level of self-esteem. In addition, limitations and practical applications of the findings were discussed.
\end{abstract}

Keywords: Perceived Chronic Social Adversity, Perceived Social Support, Depression, Self-esteem, Mediation

\section{Introduction}

Depression is a risk factor for self-injury and suicide among college students, and it can lead to many psychosomatic disorders. Students in China are mostly commonly affected by major depressive disorder [1]. A meta-analysis indicated that the prevalence of depression among college students in China is $31.38 \%$, and it shows an accelerating trend in the past decade [2].

According to Beck's cognitive theory of depression [3], individuals' cognitive evaluations of their life situations are an important determinant of the experience of subsequent specific emotions. Individuals who faced the setback of these issues may experience excessive stress, and long-term exposure to unfavorable social encounters (e.g., dishonesty, unequal treatment, being ignored, discrimination, bullying, or unemployment) may result in psychiatric problems. Research has shown that perceived chronic social adversity (PCSA) is significantly positively related to depression [4]. PCSA mainly includes three types of negative social events: social exclusion or alienation, being controlled in a close family or working relationship, and weakness in social competition [4].

Furthermore, one of the most important indicators of positive interpersonal relationships is social support. It can be conceptualized and measured in two general ways: objectively and subjectively [5]. Objective social support can be measured by instrumental support received, the size of the social network, and the frequency and extent of social contact. 
Subjective social support, also called perceived social support (PSS), is defined as individuals' perception for the quality and amount of support received from close relationships [7]. Previous research shows that PSS is much more related to depressive symptoms than objective social support [9-11]. Numerous research revealed that PSS could significantly reduce depression [8]. However, little research has addressed the effects of PCSA and PSS on other variables, including the relation between these two variables, and depression and the mediating mechanisms. Therefore, knowing the mediating mechanism between PCSA, PSS and depression has significant theoretical and practical implications.

Rosenberg defines self-esteem as an individual's overall sense of worthiness and the fundamental inner strengths of self-awareness and resistance [12]. Chronic social adversity is a strain that adversely affects an important aspect of an individual's identity. Such difficulties will be seen as substantial losses and are high-risk and challenging to one's self-worth. According to the Self-Esteem Theory, a key risk factor for depression is low self-esteem.

Despite considerable research demonstrating the effect of PSS and PCSA on college students' depression, few studies investigated the mediating effect of self-esteem in the relation between PSS and depression and the relation between PCSA and depression among Chinese college students. To fill these knowledge gaps, we used large samples in this study. We hypothesized that: (1) the level of PCSA is positively associated with depression, (2) the level of PSS is negatively associated with depression, (3) self-esteem will mediate the relation between PCSA and depression, and (4) self-esteem will mediate the relation between PSS and depression among Chinese college students.

\section{Literature Review}

\subsection{Perceived Chronic Social Adversity, Perceived Social Support and Depression}

According to the perspectives of evolutionary psychology [6], maintaining positive interpersonal relationships is a major issue for individuals. Several studies suggest that social support can help people obtain positive psychological outcomes and avoid the injury of negative emotions. Additionally, previous research has found that PSS was significantly associated with subject well-being [13]. Tucker et al. revealed that social support is an important factor in predicting the occurrence of posttraumatic reactions [14]. People with high levels of perceived social support reported low levels of PTSD [15].

In contrast with PSS, PCSA described in individuals who experience significant negative social encounter (e.g., abuse and undermining) often report higher levels of negative emotion. These events repeatedly occur, and accumulate during social interactions and competitions that people perceive as stressful or overwhelming [4]. PCSA has been discovered to be associated with a variety of long-term negative effects on emotional health (e.g., depression) [16, 17].

In this study, depression is a continuous variable (i.e., different levels of depressive affect among college students) instead of a dichotomous (clinical) variable. According to cognitive theorists, various forms of psychopathology are maintained and developed through social information processing, particularly in the construal process [18]. Based on Beck's theory, cognitions are a major determinant of an individual's feelings, and negative cognitive evaluations enhance the individual's feelings of unworthiness [19, 20]. The effects of cognitions on mental health are increasing attention in research. The findings of a meta-analysis indicated that, on average, PSS was more strongly negatively correlated with depression [21]. In addition, previous research has shown that perceptions of negative social situations (e.g., PSCA) can lead to low level mental health [22]. Based on the literature review, we speculated that higher evaluations of PSCA are related to higher levels of depression, in contrast, higher PSS is related to lower levels of depression among Chinese college students.

\subsection{The Mediating Effect of Self-esteem}

Self-esteem also plays a vital role in college students' mental health, especially in the manifestation of depression $[23,24]$. Feeling worthless is one of the emotional reactions to disability. [25]. A study conducted by Zhang et al. revealed that PCSA may result in the perception of injustice, disrespect, inferiority, low self-esteem, or worthlessness. [4]. According to theoretical work, social support is important in determining self-esteem [26]. Perception of acceptance from others is demonstrated to be associated with increased self-esteem [27]. A considerable amount of empirical research found that one's level of self-esteem may significantly be predicted by PSS [28, 29]. Based on the literature review, PSS promotes mental health by developing self-concept in the individual [30].

According to the Self-Esteem Theory of Depression, adolescents with a low level of self-esteem will feel weak about themselves, leading to more negative effect. Thus, a low level of self-esteem is associated with a high level of depression [31]. Additionally, previous studies substantiate that self-esteem are negatively related to depression. Previous studies demonstrated that the relation between self-esteem and depression was significant and negative. Results of a study, which used meta-analysis, found that self-esteem negatively predicts depression [32]. Various empirical studies support the finding $[33,34]$. Furthermore, Wang et al. reveal that a low level of self-esteem could predict a high level of depression among Chinese adults [35].

Despite plenty of empirical research in Western countries, have found that self-esteem has played an instrumental role in the relation between PSS and mental health [36-38]. However, there are conflicting arguments about whether the effect of mediation existed in Chinese college students. Chinese culture advocates good social relationships, which is related to self-concept and depression [40]. In the context of 
Confucian doctrine, self-concept may not be significant in the relation between PSS and depression. These values lead to a set of culturally unique features (e.g., high peer control), leading to individuals' low level of self-esteem [41-42]. The low level of self-esteem reported by Chinese people may lead to the mediating effect of self-esteem weakening in the relation between PSS and depression among Chinese college students.

\section{Methods}

\subsection{Procedure and Participants}

A total of 805 undergraduate students ( $65.6 \%$ females) were recruited from a college in Beijing located in northern China. They were between 17 and 28 years old $(M=20.17$ years, $\mathrm{SD}=1.46)$. We asked participants to complete an online self-report instrument on their PCSA, PSS, self-esteem, depression, and demographic background. In addition, to protect students' privacy, the survey was conducted anonymously. Participants were assured of strict confidentiality of the collected data and that only research personnel had access to the questionnaires.

\subsection{Measures}

\subsubsection{Perceived Chronic Social Adversity}

PCSA was measured by the Perceived Chronic Social Adversity Questionnaire [4], which include 28 items (e.g., "Always being rejected"). Each item was rated on a 5-point Likert-type scale from 1 (strongly disagree) to 5 (strongly agree). Higher scores indicated higher levels of PCSA. This scale has shown good psychometric properties [4]. The Cronbach's alpha coefficient for this scale was 0.98 in this study.

\subsubsection{Perceived Social Support}

PSS was measured by Multidimensional Scale of Perceived Social Support [44], which include 12 items (e.g., "I get the emotional help and support I need from my family"). Each item was rated on a 7-point Likert-type scale from 1 (very strongly dis-agree) to 7 (very strongly agree). The MSPSS focuses on three main subscales, including family, friends, and significant others. In this study, we modified some expression to fit college students. A composite score was computed and higher scores indicated higher levels of PSS. The Chinese version of this scale has shown good psychometric properties [45]. The Cronbach's alpha coefficient for this scale was 0.97 in this study.

\subsubsection{Self-esteem}

Self-esteem was measured by Rosenberg Self-Esteem Scale [12], which include 10 items (e.g., "On the whole I am satisfied with myself"). Each item was rated on a 4-point Likert-type scale from 1 (strongly disagree) to 4 (strongly agree). A composite score was computed and higher scores indicated higher levels of self-esteem. This scale has shown good psychometric properties [46]. The Cronbach's alpha coefficient for this scale was 0.79 in this study.

\subsubsection{Depression}

Depression was measured by the depression subscale of Chinese version of the short Depression Anxiety Stress Scale (DASS-21) [47], which consisted of 7 self-report items (e.g., "I felt downhearted and blue"). Each item was rated on a 4-point Likert-type scale from 0 (does not apply to me at all) to 3 (applies to me most of the time). Higher scores indicated higher levels of depression. This subscale has shown good psychometric properties [48]. The Cronbach's alpha coefficient for this subscale was 0.92 in this study.

\subsubsection{Covariates}

Previous studies have also shown that some demographic variables, such as gender, grade, and pocket money are significantly related to depression [49-51]. Therefore, students' gender $(1=$ male, $2=$ female $)$, grade, and pocket money were included in the analyses as covariates. Item for the level of pocket money incorporated seven alternative categories from 1 (0-500 Yuan) to 7 (over 3000 Yuan).

\subsection{Data Analysis}

Several data analyses were conducted to examine the relationships among PCSA, PSS, self-esteem, and depression. The data analysis followed four steps. First, Harman's one-factor test examined the common method variance [52]. Second, descriptive statistics and Pearson correlations were analyzed by SPSS 21.0. Third, the model 4 in the PROCESS macro was tested the mediation effect [53]. Bootstrapping, employing 1000 samples, was used to testing the significance of the mediated effects and to produce bias corrected percentile confidence intervals. If the $95 \%$ confidence interval for the indirect effect estimate did not include zero, the indirect effect was statistically significant at the 0.05 level. Furthermore, the size of the mediating effect was also calculated.

\section{Results}

\subsection{Assessment of Common Method Variance}

To address the issue of common method variance (CMV), we conducted a Harman's one-factor test before testing the hypotheses [49], which used exploratory factor analysis (EFA) to determine the extent of CMV in our data. In this procedure, all variables in the study are entered into an EFA. If a substantial amount of common method variance is present, either a single factor will emerge from the factor analysis, or one general factor will account for most of the variance $(>40 \%)$. In this study, the EFA resulted in seven factors with eigenvalues greater than one, and the first factor accounted for $39.52 \%$ of the total variance. The results indicated that common method variance was not a major concern in this study.

\subsection{Descriptive Analysis}

Table 1 presents the descriptive statistics for all study variables, and correlations. PSS was significantly and positively related to self-esteem but negatively related to depression. PCSA was significantly and negatively related to 
every dimension of self-esteem but positively related to depression. Self-esteem was significantly and negatively related to depression. These results supported hypotheses 1 and 2. The results also showed that PCSA and PSS were related to students' gender. PSS and self-esteem were related to pocket money; The grade was not related to every variable. Thus, we controlled gender and pocket money in the subsequent analysis.

\subsection{Examining the Mediation Model}

A mediational analysis was performed to test our third and fourth hypothesis that self-esteem would play a mediating role between PCSA and depression as well as between PSS and depression (See Figure 1). The total effect (c1) between PCSA and depression was statistically significant and positive $(\beta=0.62, p<0.001)$. The effect of PCSA on self-esteem was also significant and negative $(\mathrm{a} ; \beta=-0.32, p$ $<0.001$ ), and the effect of self-esteem on depression was significant and negative ( $\mathrm{b} ; \beta=-0.25, p<0.001)$. The size of the mediating effect $(\mathrm{a} \times \mathrm{b})$ of PCSA on depression through self-esteem was also significant $(\beta=0.08,95 \% \mathrm{CI}$ is 0.01 from to 0.02 ).

Table 1. Descriptive statistics and correlations among variables $(N=805)$.

\begin{tabular}{llllllll}
\hline Variable & M & SD & $\mathbf{1}$ & $\mathbf{2}$ & $\mathbf{3}$ & $\mathbf{4}$ \\
\hline PCSA & 60.70 & 26.24 & - & & & \\
PSS & 58.40 & 16.04 & $-0.15^{* * *}$ & - & & \\
Self-esteem & 28.20 & 5.18 & $-0.32^{* * *}$ & $0.45^{* * *}$ & - & - & \\
Depression & 4.16 & 4.78 & $0.62^{* * *}$ & $-0.15^{* * *}$ & $-0.42^{* * *}$ & - & -0.06 \\
Gender & 1.66 & 0.48 & $-0.10^{* *}$ & $0.07^{*}$ & 0.04 & - & -0.05 \\
Grade & 3.06 & 0.80 & 0.05 & -0.06 & -0.07 & -0.029 \\
Pocket money & 3.18 & 1.32 & 0.05 & $0.12^{* *}$ & $0.08^{*}$ & -0.03 & -0.054 \\
\hline
\end{tabular}

${ }^{1}$ Note. $* p<0.05 ; * * p<0.01 ; * * * p<0.001$.

${ }^{2}$ Skewness (rang from -0.66 to 1.41 ) and kurtosis (rang from -1.57 to 1.64 ) of all variables in this study are satisfied the normality criteria (skewness $<3$, kurtosis $<10$; Kline, 2010).

The total effect (c2) between PSS and depression was statistically significant and negative $(\beta=-0.15, p<0.001)$. The effect of PSS on self-esteem was also significant and positive $(a ; \beta=0.44, p<0.001)$, and the effect of self-esteem on depression was significant and negative $(\mathrm{b} ; \beta=-0.43, p<$ $0.001)$. The size of the mediating effect $(\mathrm{a} \times \mathrm{b})$ of PSS on depression through self-esteem was also significant $(\beta=$

\section{$-0.19,95 \% \mathrm{CI}$ is -0.24 from to -0.15$)$.}

Finally, the direct effects (c1') of PCSA on depression was significant $(\beta=0.54, p<0.001)$, but the direct effects (c2') of PSS on depression was nonsignificant $(\beta=0.03, p=0.36$ ), which indicated self-esteem played a partial mediating role between PCSA and depression, whereas a total mediating role between PSS and depression.

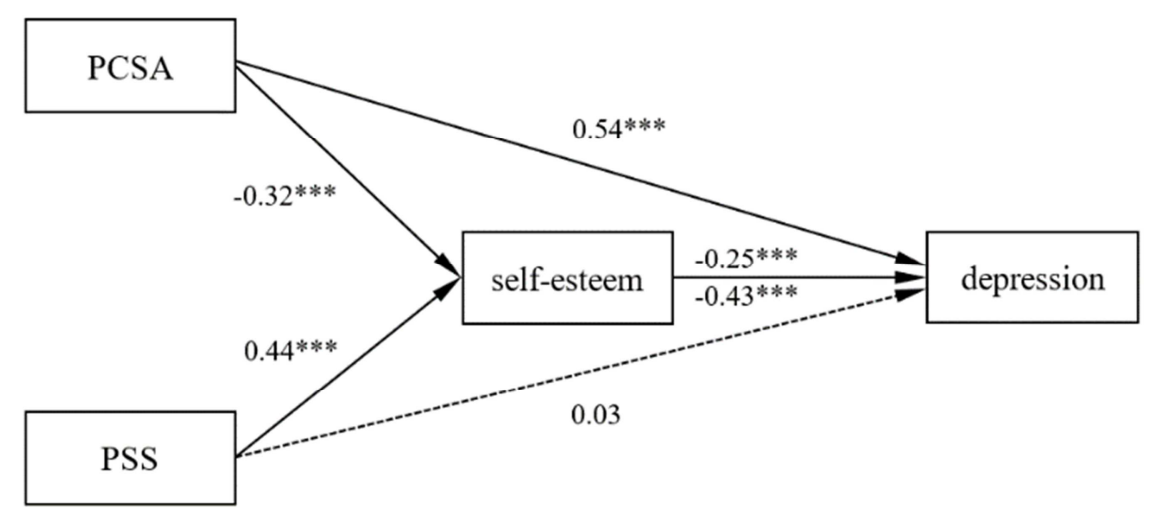

Notes. ${ }^{* * *} \mathrm{p}<0.001$; In order to simplify the model, other no significant control variables are not shown.

Figure 1. The mediation model predicting Chinese college students'depression from PCSA and PSS respectively (N =805).

\section{Discussion}

We principally investigated the critical role of self-esteem in the relation between Chinese college students' cognition of social encounter (i.e., PCSA and PSS) and depression. The primary findings of this study were twofold. First, PCSA was significantly positively related to depression, whereas PSS was significantly negatively related to depression among Chinese college students. Second, A partial mediating effect of self-esteem manifested in the relation between PCSA and depression and the relation between PSS and depression. Thus, the results supported the hypothesized mediation model.

\subsection{Perceived Chronic Social Adversity, Perceived Social Support and Depression}

As hypothesized, PCSA was significantly positively related to depression among Chinese college students. Our result was 
consistent with Beck's theory. Cognitions constitute a significant determinant of an individual's feelings. Negative cognitive evaluations enhance the individual's feelings of unworthiness, while positive cognitive evaluations enhance subjective well-being and other positive affect [19, 20]. More specifically, positive cognition of social encounters will reduce the person's level of depression, but negative cognition of social encounters will intensify the level of depression. According with previous research, PSS was significantly and negatively related to depression among Chinese people [54]. To the contrary, several studies have suggested a positive relation between negative social processes (e.g., unfair treatment and deception) and negative emotion (e.g., depression and shame) [55-56].

\subsection{Mediational Role of Self-esteem}

The notion that PCSA and PSS directly relate to depression has gained considerable theoretical and empirical support [57, 58]. However, questions regarding the underlying mediating mechanisms have not been addressed fully. Consistent with our hypothesis, self-esteem play a mediated role in the relations between perceived social encounter (i.e., PCSA and PSS) and depression among Chinese college students.

More specifically, PSS promotes self-esteem by conveying messages to individuals that they are loved, valued, and cared for by other people, as well as by fostering belonging to a community [59, 60]. In turn, a high level of self-esteem lowers the possibility of experiencing negative emotion (e.g., depression) [61]. Moreover, several researchers have found the effect of PSS on self-esteem. Ikiz and Cakar found that people who perceive social support from others report a high level of self-esteem [62]. Furthermore, research about Iran university students reveal that PSS from families were predictive variables for self-esteem [63]. In line with the definition, PCSA focuses on people's subjective evaluation of negative social events (e.g., deception, unfair treatment, emotional blackmail) during social interactions and social competition [4]. According to the sociometer theory [16], individuals whom have been rejected by others will underestimate themselves. Self-esteem plays the role of sociometer, which can measure individuals' social acceptance. As a result, social exclusion or rejection will have a negative effect on self-esteem, signaling to the individual that they have experienced a traumatic event that has jeopardized their ability to fulfill the need to belong. Furthermore, people who are weak in social completion may experience unequal treatment, disrespect, inferiority, low self-esteem, or feelings of worthlessness. Blackhart et al. revealed that rejection by close friends and family may lead to a greater reduction in affect and self-esteem than rejection by strangers or acquaintances [64]. Moreover, unlike previous research about self-esteem among Chinese people, we did not find that the effect of perceived social encounters on self-esteem was discounted. Two possible reasons may explain this result: (1) "One Child Policy" make the population of children in a family significantly decline, and (2) the number of nuclear families remained stable with a steady rise [65]. The reason led to Chinese young people not strongly approving of Chinese traditional values. Thus, we did not find a significant deference from Western young people in the effect of perceived social encounters on self-esteem.

According with the Self-Esteem Theory of Depression [66], self-esteem is one of the most critical factors contributing to students feeling good about themselves and reducing depression risk. The negative relation between self-esteem and depression is consistent with previous studies [33]. The results of our research provide evidence that self-esteem acts as a protective factor against depression. Traumatic events are less likely to threaten individuals with high level of self-esteem [67]. In general, the results of our study have shed new light upon psychological mechanisms related to depression.

\subsection{Implications}

It is critical to prevent psychological difficulties at an early stage. Our theoretical findings in this study may provide a helpful framework for understanding what contributes to psychological difficulty, particularly depression, in Chinese college students. Given that PCSA and PSS act as significant predictors of the emotional health of Chinese college students, colleges should make systematic efforts to promote students' positive evaluation of their social encounters in order to promote their self-esteem and reduce the possibility of depression. Additionally, we discovered that self-esteem mediated the relation between PCSA and depression, and between PSS and depression among Chinese college students. Hence, a high level of self-esteem is beneficial to students who suffer from depression. As such, the mental health practitioner should pay more attention to self-esteem when working with students with depression. Colleges should provide effective psychological intervention services and take advantage of the mental health class to promote students' self-esteem and other positive traits, not just focusing on their study performance and employment rate. In China, most colleges have a center for mental health, and every 200 students should have one tutor to provide mental health and career development service. A variety of mental health services are available for students with psychological and emotional problems in colleges. Based on the results, depression prevention should improve in Chinese colleges through mental health care. To reduce the risk of students developing depression, we proposed carrying out programs of intervention (e.g., cognitive-behavioral therapy and interpersonal-process therapy) in the classroom that teaches positive cognition strategies to deal with social encounters.

\subsection{Limitations and Future Direction}

Despite the implications of the theories and practices discussed above, several limitations in our study have should be addressed. First, this study is a correlational study and we cannot make any causal conclusions from the current results. 
In future studies, longitudinal or experimental designs should be used to overcome this limitation. Second, all variables in this study were measured via self-report of the college students. Self-reporting may inflate findings through common method variance [52]. However, in order to minimize common method bias, we took several preparatory steps, including providing intelligible scales to measure all variables, stressing the importance of the study to participants, and testing a one-factorial model against the theoretical model with CFA. Nonetheless, in the future, we recommend the use of multiple data sources and multiple time points when collecting data for research. Third, this study did not control possible context and individual variables, such as positivity, problem-focused coping, and perfectionism, which are important factors influencing students' depression. There should be more research on the effects of context and other individual difference variables on the relations among PCSA, PSS, self-esteem, and depression.

\section{Conclusion}

We investigated the underlying psychosocial mechanisms accounting for the association between cognition of social encounters (i.e., PCSA and PSS) and depression in Chinese college students. Although many studies are needed before drawing any definitive conclusions, we found that PSS and self-esteem negatively predicted depression. Additionally, they play a significant role in decreasing depression among Chinese college students. While PCSA positively predicted depression, that means PSCA is a risk factor for college students' mental health. Furthermore, we propose that understanding depression cannot be undertaken by only examining cognition of positive and negative social encounters (e.g., PCSA and PSS). Instead this cognition must combine with other psychological variables, enhancing their individuals' mental health. The current study also showed that self-esteem plays a mediating role respectively in the relation between PCSA and depression, and the relation between PSS and depression. Increasing self-esteem can play significant role in decreasing depression of college students. In general, teachers and counselors should focus more on increasing students' self-esteem, particularly those with unpleasant social interactions, to alleviate depression among college students.

\section{Acknowledgements}

This study was not funded. The authors thanked all the participants for their participation in the study.

\section{References}

[1] Yang, X., Jiang, C., Pan, W., X, W., Liang, F., L, N., \& Pan, G. (2014). Prevalence of psychiatric disorders among children and adolescents in northeast China. PLoS One, 9 (10), 1-9. doi: 10.1371/journal.pone.0111223.
[2] Wang, M., Liu, J., Wu, X., Li, L., Hao, X., Shen, Q., Huang, M., \& Sun, R. (2020). The prevalence of depression among students in Chinese universities over the past decade: A meta-analysis. Journal of Hainan Medical University, 26 (9), 52, 59-65. doi: 10.13210/j.cnki.jhmu.20200218.001.

[3] Beck, A. T. (1993). Cognitive therapy: past, present, and future. Journal of consulting and clinical psychology, 61 (2), 194-198. doi: 10.1037/0022-006X.61.2.194.

[4] Zhang, J., Ding, C., Tang, Y., Zhang, C., \& Yang, D. (2017). A measure of perceived chronic social adversity: development and validation. Frontiers in psychology, 8, 2168. doi: 10.3389/fpsyg.2017.02168.

[5] George, L. K. (1996). Social factors and illness. In R. H. Binstock, L. K. George, V. W. Marshall, G. C. Myers, \& J. H. Schulz (Eds.), Handbook of aging and the social sciences. Academic Press.

[6] Kanazawa, S., \& Savage, J. (2009). An evolutionary psychological perspective on social capital. Journal of Economic Psychology, 30 (6), 873-883. doi: 10.1016/j.joep.2009.08.002.

[7] Landerman, R. (1999). EPESE Composite Measures and Other Commonly Used Measures: Description and Documentation of Original Variables and Variables with Missing Values Imputed. Durham, NC: The Center on Aging and Human Development, Duke University Medical Center.

[8] Commerford, M. C., \& Reznikoff, M. (1996). Relationship of religion and perceived social support to self-esteem and depression in nursing home residents. Journal of Psychology, 130 (1), 35-50. doi: 10.1080/00223980.1996.9914986.

[9] Blazer, D. G. (1982). Social support and mortality in an elderly community population. American journal of epidemiology, $115 \quad$ (5), 684-694. doi: 10.1093/oxfordjournals.aje.a113351.

[10] George, L. K., Blazer, D. G., Hughes, D. C., \& Fowler, N. (1989). Social support and the outcome of major depression. The British Journal of Psychiatry, 154 (4), 478-485. doi: 10.1192/bjp.154.4.478.

[11] Turner, R. J., \& Noh, S. (1988). Physical disability and depression: A longitudinal analysis. Journal of health and social behavior, 29 (1), 23-37. doi: 10.2307/2137178.

[12] Rosenberg, M. (1965). Rosenberg self-esteem scale (RSE). Acceptance and commitment therapy. Measures package, 61 (52), 18

[13] Fikret, G. (2010). The effect of perceived social support on subjective well-being. Procedia - Social and Behavioral Sciences, 2 (2), 3844-3849. doi: 10.1016/j.sbspro.2010.03.602.

[14] Tucker, P., Pfefferbaum, B., Nixon, S. J., \& Dickson, W. (2000). Predictors of post-traumatic stress symptoms in Oklahoma City: Exposure, social support, peri-traumatic responses. The Journal of Behavioral Health Services \& Research, 27 (4), 406-416. doi: 10.1007/bf02287822.

[15] Ozer, E. J., Best, S. R., Lipsey, T. L., \& Weiss, D. S. (2003). Predictors of posttraumatic stress disorder and symptoms in adults: A meta-analysis. Psychological Bulletin, 129, 52-73. doi: 10.1037/0033-2909.129.1.52. 
[16] Baumeister, R. F., \& Leary, M. R. (1995). The need to belong: Desire for interpersonal attachments as a fundamental human motivation. Psychological Bulletin, 117 (3), 497-529. doi: 10.1037/0033-2909.117.3.497.

[17] Macdonald, G., \& Leary, M. (2005). Roles of Social Pain and Defense Mechanisms in Response to Social Exclusion: Reply to Panksepp (2005) and Corr (2005). Psychological Bulletin, 131 (2), 237-240. doi: 10.1037/0033-2909.131.2.237.

[18] Crick, N. R., \& Dodge, K. A. (1994). A review and reformulation of social information processing mechanisms in children's social adjustment. Psychological Bulletin, 115 (1), 74-101. doi: 10.1037/0033-2909.115.1.74.

[19] Rush, A. J., \& Beck, A. T. (1978). Cognitive therapy of depression and suicide. American Journal of Psychotherapy, 32 (2), 201-219. doi: 10.1176/appi.psychotherapy.1978.32.2.201.

[20] Beck, A. T., \& Alford, B. A. (2009). Depression: Causes and treatment. University of Pennsylvania Press.

[21] Yang, C. X., Zhang, D. J., Liang, Y. H., \& Tian-Qiang, H. U. (2016). Relationship between social support and depression among college students in China: a meta-analysis. Chinese Mental Health Journal, 30, 939-945.

[22] Isaksson, J., Nilsson, K. W., \& Lindblad, F. (2013). Early psychosocial adversity and cortisol levels in children with attention-deficit/hyperactivity disorder. European Child \& Adolescent Psychiatry, 22 (7), 425-432. doi: 10.1007/s00787-013-0383-0.

[23] Gardner, A. A., \& Lambert, C. A. (2019). Examining the interplay of self-esteem, trait-emotional intelligence, and age with depression across adolescence. Journal of adolescence, 71, 162-166. doi: 10.1016/j.adolescence.2019.01.008.

[24] Yalçınkaya-Alkar, Ö. (2020). Is self-esteem mediating the relationship between cognitive emotion regulation strategies and depression. Current Psychology, 39, 220-228. https: //doi.org/10.1007/s12144-017-9755-9.

[25] Bruce, M. L. (2001). Depression and disability in late life: directions for future research. The American Journal of geriatric psychiatry, 9 (2), 102-112. doi: 10.1097/00019442-200105000-00003.

[26] Arslan, C. (2009). Anger, self-esteem, and perceived social support in adolescence. Social Behavior and Personality: an international journal, 37 (4), 555-564. doi: 10.2224/sbp.2009.37.4.555.

[27] Lachowicz-Tabaczek, K., \& J Niecińska. (2011). Self-concept and self-esteem: how the content of the self-concept reveals sources and functions of self-esteem. Polish Psychological Bulletin, 42 (1), 24-35. doi: 10.2478/v10059-011-0005-y.

[28] Hoffman, M. A., Levy-Shiff, R., \& Ushpiz, V. (1993). Moderating effects of adolescent social orientation on the relation between social support and self-esteem. Journal of Youth \& Adolescence, 22 (1), 23-31. doi: 10.1007/BF01537901.

[29] Feng, K., \& You, X. (2013). Loneliness and self-esteem as mediators between social support and life satisfaction in late adolescence. Social Indicators Research, 110 (1), 271-279. doi: 10.1007/s11205-011-9930-6.

[30] Kim, J., \& Nesselroade, J. (2003). Relationships among social support, self-concept, and wellbeing of older adults: A study of process using dynamic factor models. International journal of behavioral development, 27 (1), 49-65. doi: $10.1080 / 01650250244000010$.

[31] Orth, U., Robins, R. W., \& Roberts, B. W. (2008). Low self-esteem prospectively predicts depression in adolescence and young adulthood. Journal of Personality \& Social $\begin{array}{llll}\text { Psychology, } & 95 & \text { (3), } & 695-708 .\end{array}$ 10.1037/0022-3514.95.3.695.

[32] Sowislo, J. F., Orth, U., Meier, L. L. (2014). What constitutes vulnerable self-esteem? comparing the prospective effects of low, unstable, and contingent self-esteem on depressive symptoms. Journal of Abnormal Psychology, 123 (4), 737-53. doi: $10.1037 / \mathrm{a} 0037770$.

[33] Bajaj, B., Robins, R. W., Pande, N. (2016). Mediating role of self-esteem on the relationship between mindfulness, anxiety, and de-pression. Personality \& Individual Differences, 96, 127-131. doi: 10.1016/j.paid.2016.02.085.

[34] Cha, Meehee. (2016). The mediation effect of mattering and self-esteem in the relationship between socially prescribed perfectionism and depression: based on the social disconnection model. Personality \& Individual Differences, 88, 148-159. doi: 10.1016/j.paid.2015.09.008.

[35] Wang, X., Wang, W., Xie, X., Wang, P., Wang, Y., \& Jia, N. (2018). Self-esteem and depression among chinese adults: a moderated mediation model of relationship satisfaction and positive affect. Personality and Individual Differences, 135, 121-127. doi: 10.1016/j.paid.2018.06.055.

[36] Saltzman, K. M., Holahan, C. J. (2015). Social support, self-efficacy, and depressive symptoms: an integrative model. Journal of Social \& Clinical Psychology, 21 (3), 309-322. doi: 10.1521/jscp.21.3.309.22531.

[37] Symister, P., Friend, R. (2003). The influence of social support and problematic support on optimism and depression in chronic illness: a prospective study evaluating self-esteem as a mediator. Health Psychology, 22 (2), 123-129. https: //DOI: 10.1037/0278-6133.22.2.123.

[38] Yarcheski, A., Mahon, N. E., Yarcheski, T. J. (2001). Social support and well-being in early adolescents: the role of mediating variables. Clinical Nursing Research, 10 (2), 163-181. doi: 10.1177/C10N2R6.

[39] Chen, J. K., Avi Astor, R. (2009). The perpetration of school violence in Taiwan: An analysis of gender, grade level and school type. School Psychology International, 30 (6), 568-584. doi: $10.1177 / 0143034309107076$.

[40] Dang, Q. X., Ying, L. I., Zhang, B. S. (2016). Interpersonal relationships and adolescent depression: roles of self-esteem and gender. Chinese Journal of Clinical Psychology, 24 (1), 69-73, 80. doi: 10.16128/j.cnki.1005-3611.2016.01.016.

[41] Jiang, Q., D'Apolito, R. (1999). A reconsideration of social control and differential association theories: an empirical investigation of parental and peer influences on delinquency. Free Inquiry in Creative Sociology, 27 (2), 73-92.

[42] Milevsky, A., Schlechter, M., Netter, S., Keehn, D. (2007). Maternal and paternal parenting styles in adolescents: Associations with self-esteem, depression and life-satisfaction. Journal of Child And Family Studies, 16 (1), 39-47. doi: 10.1007/s10826-006-9066-5. 
[43] Leung, C. H., Choi, E. (2010). A Qualitative Study of Self-Esteem, Peer Affiliation, and Academic Outcome among Low Achieving Students in Hong Kong. New horizons in education, 58 (1), 22-42.

[44] Zimet, G. D., Powell, S. S., Farley, G. K., Werkman, S., Berkoff, K. A. (1990). Psychometric characteristics of the multidimensional scale of perceived social support. Journal of personality assessment, 55 (3), 610-617. doi: $10.1080 / 00223891.1990 .9674095$.

[45] Xia, M., Yang, C. (2019). The relationship among social support, self-esteem, affect balance and loneliness in individuals with substance use disorders in China. Journal of community psychology, 47 (5), 1269-1281. doi: 10.1002/jcop. 22190 .

[46] Chen, W., Niu, G. F., Zhang, D. J., Fan, C. Y., Tian, Y., \& Zhou, Z. K. (2016). Socioeconomic status and life satisfaction in Chinese adolescents: Analysis of self-esteem as a mediator and optimism as a moderator. Personality and Individual Differences, 95, 105-109. doi: 10.1016/j.paid.2016.01.036.

[47] Moussa, M. T., Lovibond, P. F., Laube, R. (2001). Psychometric properties of a Chinese version of the 21-item depression anxiety stress scales (DASS21). Sydney, NSW: Transcultural Mental Health Centre. Cumberland Hospital.

[48] Lu, S., Hu, S., Guan, Y., Xiao, J., Cai, D., Gao, Z., Margraf, J. (2018). Measurement invariance of the Depression Anxiety Stress Scales-21 across gender in a sample of Chinese university students. Frontiers in psychology, 9, 2064. doi: 10.3389/fpsyg.2018.02064.

[49] Perry, Nagin, Anthony, Salandy, Angela, Diaz. (2019). Unpacking the influence of abuse and depression on grades among urban ethnic minority adolescents and young adults. Annals of global health, 85 (1), 55. doi: 10.5334/aogh.2478.

[50] Rudolph, K. D., Flynn, M. (2007). Childhood adversity and youth depression: Influence of gender and pubertal status. Development and psychopathology, 19 (2), 497-521. doi: 10.1017/S0954579407070241.

[51] Tesfaye, G., Derese, A., Hambisa, M. T. (2014). Substance use and associated factors among university students in Ethiopia: a cross-sectional study. Journal of addiction, 2014, 1-8. doi: $10.1155 / 2014 / 969837$.

[52] Podsakoff, P. M., Organ, D. W. (1986). Self-reports in organizational research: Problems and prospects. Journal of management, 12 (4), 531-544. doi: $10.1177 / 014920638601200408$.

[53] Hayes, A. F. (2013). "An introduction to mediation, moderation, and conditional process analysis: A regression-based approach", New York, NY: Guilford Press.

[54] Liang, D., Teng, M., Xu, D. (2019). Impact of perceived social support on depression in Chinese rural-to-urban migrants: The mediating effects of loneliness and resilience. Journal of community psychology, 47 (7), 1603-1613. doi: 10.1002/jcop.22215.

[55] Woo, B., Wang, K., Falcón, L. M. (2020). Unfair treatment, coping strategies, and depression among Puerto Ricans in Boston. Cultural Diversity and Ethnic Minority Psychology, 26 (2), 229-238. doi: 10.1037/cdp0000291.
[56] Seiter, J. S., Bruschke, J. (2007). Deception and Emotion: The Effects of Motivation, Relationship Type, and Sex on Expected Feelings of Guilt and Shame Following Acts of Deception in United States and Chinese Samples. Communication Studies, 58 (1), 1-16. doi: $10.1080 / 10510970601168624$.

[57] Ding, C., Zhang, J., D Yang. (2018). A pathway to psychological difficulty: perceived chronic social adversity and its symptomatic reactions. Frontiers in Psychology, 9, 615. doi: $10.3389 /$ fpsyg.2018.00615.

[58] Hosseini, F. S., Sharifi, N., Jamali, S. (2021). Correlation anxiety, stress, and depression with perceived social support among the elderly: a cross-sectional study in Iran. Ageing International, $46 \quad$ (1), 108-114. doi: 10.1007/s12126-020-09376-9.

[59] Lakey, B., Cohen, S. (2000). Social support theory and measurement. In S. Cohen, L. G. Underwood, \& B. H. Gottlieb (Eds.), Social support measurement and intervention: A guide for health and social scientists. Oxford University Press. doi: 10.1093/med: psych/9780195126709.003.0002.

[60] Turner, R. J., Turner, J. B., Hale, W. B. (2014). Social relationships and social support. In Sociology of mental health. Springer, Cham.

[61] Lee, C., Dickson, D. A., Conley, C. S., Holmbeck, G. N. (2014). A closer look at self-esteem, perceived social support, and coping strategy: A prospective study of depressive symptomatology across the transition to college. Journal of Social and Clinical Psychology, 33 (6), 560-585. doi: 10.1521/jscp.2014.33.6.560.

[62] Ikiz, F. E., Cakar, F. S. (2010). Perceived social support and self-esteem in adolescence. Procedia-Social and Behavioral Sciences, 5, 2338-2342. doi: 10.1016/j.sbspro.2010.07.460.

[63] Naseri, L., Mohamadi, J., Sayehmiri, K., Azizpoor, Y. (2015). Perceived Social Support, Self-Esteem, and Internet Addiction Among Students of Al-Zahra University, Tehran, Iran. Iranian Journal of Psychiatry and Behavioral Sciences, 9 (3), e421. doi: 10.17795/ijpbs-421.

[64] Blackhart, G. C., Nelson, B. C., Knowles, M. L., Baumeister, R. F. (2009). Rejection Elicits Emotional Reactions but Neither Causes Immediate Distress nor Lowers Self-Esteem: A Meta-Analytic Review of 192 Studies on Social Exclusion. Personality and Social Psychology Review, 13 (4), 269-309. doi: $10.1177 / 1088868309346065$.

[65] Yuesheng, W. (2020). Analysis of the Changes in Rural Chinese Family Structure since Reform and Opening Up. Contemporary Social Sciences. 1, 102-124. doi: 10.19873/j.cnki.2096-0212.2020.01.008.

[66] Orth, U., Robins, R. W. (2013). Understanding the link between low self-esteem and depression. Current directions in psychological science, 22 (6), 455-460. doi: 10.1177/0963721413492763.

[67] Harmon-Jones, E., Simon, L., Greenberg, J., Pyszczynski, T., Solomon, S., McGregor, H. (1997). Terror management theory and self-esteem: Evidence that increased self-esteem reduced mortality salience effects. Journal of personality and social psychology, 72 (1), 24-36. doi: 10.1037/0022-3514.72.1.24. 